\title{
RELATIONSHIP BETWEEN THE HYERS-ULAM STABILITY AND THE MOORE-PENROSE INVERSE
}

\author{
QIANGLIAN HUANG ${ }^{1 *}$ AND MOHAMMAD SAL MOSLEHIAN ${ }^{2}$
}

\begin{abstract}
In this paper, we establish a link between the Hyers-Ulam stability and the Moore-Penrose inverse, that is, a closed operator has the Hyers-Ulam stability if and only if it has a bounded Moore-Penrose inverse. Meanwhile, the stability constant can be determined in terms of the Moore-Penrose inverse. Based on this result, some conditions for the perturbed operators having the Hyers-Ulam stability are obtained and the Hyers-Ulam stability constant is expressed explicitly in the case of closed operators. In the case of the bounded linear operators we obtain some characterizations for the Hyers-Ulam stability constants to be continuous. As an application, we give a characterization for the Hyers-Ulam stability constants of the semi-Fredholm operators to be continuous.
\end{abstract}

\section{Introduction and Preliminaries}

More than a half century ago, Ulam [27] proposed the first stability problem concerning group homomorphisms, which was partially solved by Hyers [13] in the framework of Banach spaces. Later, Aoki [1] proved the stability of the additive mapping and Th.M. Rassias [24] investigated the stability of the linear mapping for mappings $f$ when the norm of the Cauchy difference $f(x+y)-f(x)-f(y)$ is bounded by the expression $\varepsilon\left(\|x\|^{p}+\|y\|^{p}\right)$ for some $\varepsilon \geq 0$ and some $0 \leq p<1$. J.M. Rassias [23] considered the same problem with $\varepsilon\left(\|x\|^{p}\|y\|^{p}\right)$. A large number of papers have been published in connection with various generalizations of HyersUlam theorem in several wide frameworks. In particular, it is nearly related to the notion of perturbation $[5,19]$ and geometry of Banach spaces [7, 26]. The interested reader is referred to books $[6,14,15,20]$ and references therein.

In 2003, Miura, Miyajima and Takahasi [17, 18] investigated the notion of the Hyers-Ulam stability of a mapping between normed linear spaces and obtained some stability results for particular linear differential operators. Takagi, Miura and Takahasi [25] considered the Hyers-Ulam stability of bounded linear operators

2010 Mathematics Subject Classification. 47A55, 46A32, 39B82, 47A05, 47A30.

Key words and phrases. Hyers-Ulam stability; Moore-Penrose inverse; generalized inverse; reduced minimum modulus; closed linear operator; $T$-boundedness; semi-Fredholm operator. 
in Banach spaces. After then, Hirasawa and Miura [9] gave some necessary and sufficient conditions under which a closed operator in a Hilbert space has the HyersUlam stability. Moslehian and Sadeghi [21] studied the Hyers-Ulam stability of $T$ bounded operators. They also discussed the best constant of Hyers-Ulam stability. In the sequel, we need some terminology.

Definition 1.1. Let $X, Y$ be normed linear spaces and let $T$ be a (not necessarily linear) mapping from $X$ into $Y$. We say that $T$ has the Hyers-Ulam stability if there exists a constant $K>0$ with the property: (a) For any $y$ in the range $R(T)$ of $T, \varepsilon>0$ and $x$ in the Domain $D(T)$ of $T$ with $\|T x-y\| \leq \varepsilon$, there exists an element $x_{0} \in D(T)$ such that $T x_{0}=y$ and $\left\|x-x_{0}\right\| \leq K \varepsilon$. We call such $K>0$ a Hyers-Ulam stability constant for $T$ and denote by $K_{T}$ the infimum of all HyersUlam stability constants for $T$. If $K_{T}$ is a Hyers-Ulam stability constant for $T$, then $K_{T}$ is called the Hyers-Ulam stability constant for $T$.

Roughly speaking, if $T$ has the Hyers-Ulam stability, then to each $\varepsilon$-approximate solution $x$ of the equation $T x=y$ there corresponds an exact solution $x_{0}$ of the equation in a $K_{\varepsilon}$-neighborhood of $x$; see [9].

Remark 1.2. [9, 21] If $T$ is linear then condition (a) is equivalent to:

(b) For any $\varepsilon>0$ and $x \in D(T)$ with $\|T x\| \leq \varepsilon$, there exists $x_{0} \in D(T)$ such that $T x_{0}=0$ and $\left\|x-x_{0}\right\| \leq K \varepsilon$.

If we denote the null space of $T$ by $N(T)$, then the condition (b) is equivalent to:

(c) For any $x \in D(T)$, there exists $x_{0} \in N(T)$ such that $\left\|x-x_{0}\right\| \leq K\|T x\|$.

Let $X, Y$ be Banach spaces. Let $L(X, Y), C(X, Y)$ and $B(X, Y)$ denote the linear space of all linear operators, the homogeneous set of all closed linear operators with a dense domain and the Banach space of all bounded linear operators from $X$ into $Y$, respectively. The identity operator is denoted by $I$. Let us introduce the reduced minimum modulus of closed linear operators.

Definition 1.3. [16] The reduced minimum modulus of $T \in C(X, Y)$ is defined by

$$
\gamma(T)=\inf \left\{\|T x\|: \quad x \in D(T) \text { with } d(x, N(T)):=\inf _{z \in N(T)} d(x, z)=1\right\} .
$$

It is easy to see that $\gamma(T)=\sup \{\alpha \geq 0:\|T x\| \geq \alpha d(x, N(T)), x \in D(T)\}$ and if $X$ and $Y$ are Hilbert spaces, then

$$
\gamma(T)=\inf \left\{\|T x\|: \quad x \in D(T) \cap N(T)^{\perp} \text { with }\|x\|=1\right\},
$$

where $\perp$ denotes the orthogonal complement in Hilbert spaces; see also [4]. 
Theorem 1.4. [9] Let $X, Y$ be Hilbert spaces and $T \in C(X, Y)$. Then $T$ has the Hyers-Ulam stability if and only if it has closed range. In this case, $K_{T}=\gamma(T)^{-1}$.

Let us introduce the notion of a generalized inverse (see e.g. [2]) and that of the Moore-Penrose inverse of a closed operator.

Definition 1.5. [2] An operator $T \in C(X, Y)$ possesses a (bounded) generalized inverse if there exists an operator $S \in B(Y, X)$ such that $R(S) \subseteq D(T)$ and (1) $T S T x=T x$ for all $x \in D(T)$; (2) STSy $=S y$ for all $y \in Y$; (3) ST is continuous. We denote a generalized inverse of $T$ by $T^{+}$.

In general, the generalized inverse need not exist and is not unique even if it exists. We need the following lemma concerning the existence of generalized inverses.

Lemma 1.6. [22] (a) Let $T \in C(X, Y)$. Suppose that $N(T)$ has a topological complement $N(T)^{c}$ in $X$ and $\overline{R(T)}$ has a topological complement $\overline{R(T)}^{c}$ in $Y$, i.e.,

$$
X=N(T) \oplus N(T)^{c} \quad \text { and } \quad Y=\overline{R(T)} \oplus \overline{R(T)}^{c} .
$$

Let $P$ denote the projector of $X$ onto $N(T)$ along $N(T)^{c}$ and $Q$ denote the projector of $Y$ onto $\overline{R(T)}$ along $\overline{R(T)}^{c}$. Then there is a unique $S \in C(Y, X)$ satisfying: 1) $T S T=T$ on $D(T) ; 2) S T S=S$ on $D(S) ; 3) S T=I-P$ on $D(T)$ and 4$)$ $T S=Q$ on $D(S)$, where $D(S)=R(T)+\overline{R(T)}^{c}$.

(b) Under the assumptions of part (a), $S$ is bounded if and only if $R(T)$ is closed. In this case, $S$ is a bounded generalized inverse of $T$ with $D(S)=Y, N(S)=R(T)^{c}$ and $R(S)=D(T) \cap N(T)^{c}$.

Definition 1.7. [22] Let $X, Y$ be Hilbert spaces and $T \in C(X, Y)$. If the topological decompositions in Lemma 1.6 are orthogonal, i.e.,

$$
X=N(T) \dot{+} N(T)^{\perp} \quad \text { and } \quad Y=\overline{R(T)} \dot{+} R(T)^{\perp},
$$

where $\dot{+}$ denotes the orthogonal direct sum, then the corresponding generalized inverse is usually called the Moore-Penrose inverse of $T$. In this case, the operators $P$ and $Q$ in Lemma 1.6 are orthogonal projectors. The Moore-Penrose inverse of $T$ is always denoted by $T^{\dagger}$.

Remark 1.8. The operator $T \in C(X, Y)$ has a generalized inverse $T^{+} \in B(Y, X)$ if and only if

$$
X=N(T) \oplus N(T)^{c} \quad \text { and } \quad Y=R(T) \oplus R(T)^{c} .
$$


In this case, it follows from the closed graph theorem that the operator $T T^{+}$is a projector from $Y$ onto $R(T)$ such that $N\left(T T^{+}\right)=N\left(T^{+}\right)$and $R\left(T T^{+}\right)=R(T)$. Meanwhile, by the condition (3) in Definition 1.5, $T^{+} T$ can be extended uniquely to a projector from $X$ onto $\overline{R\left(T^{+}\right)}$with the null space $N(T)$ and the range $\overline{R\left(T^{+}\right)}$.

It is well known that the perturbation analysis of Moore-Penrose inverses and generalized inverses in Hilbert and Banach spaces are very important in practical applications of operator theory and has been widely studied; cf. [3, 4, 8, 10, 11, 12, 22, 28, 29]. Recently, the perturbation of generalized inverses for linear operators in Hilbert spaces or Banach spaces has been studied in [4, 8, 10, 11, 12, 28, 29]. To achieve our results, we need the concept of $T$-boundedness as follows.

Definition 1.9. [16] Let $T$ and $P$ be linear operators with the same domain space such that $D(T) \subseteq D(P)$ and

$$
\|P x\| \leq a\|x\|+b\|T x\| \quad(x \in D(T))
$$

where $a, b$ are nonnegative constants. Then we say $P$ is relatively bounded with respect to $T$ or simply $T$-bounded and the greatest lower bound of all possible constants $b$ will be called the relative bound of $P$ with respect to $T$ or simply the T-bound.

Theorem 1.10. [12] Let $X, Y$ be Banach spaces and let $T \in C(X, Y)$ with a bounded generalized inverse $T^{+} \in B(Y, X)$. Let $\delta T \in L(X, Y)$ be $T$-bounded with constants $a, b$. If $a\left\|T^{+}\right\|+b\left\|T T^{+}\right\|<1$, then the following statements are equivalent: (1) $B:=T^{+}\left(I+\delta T T^{+}\right)^{-1}: Y \rightarrow X$ is a bounded generalized inverse of $\bar{T}:=T+\delta T$;

(2) $\left(I+\delta T T^{+}\right)^{-1} R(\bar{T})=R(T)$;

(3) $\left(I+\delta T T^{+}\right)^{-1} \bar{T}$ maps $N(T)$ into $R(T)$;

(4) $R(\bar{T}) \cap N\left(T^{+}\right)=\{0\}$.

Moreover, if one of the conditions above is true, then $\bar{T}$ is a closed operator and its range $R(\bar{T})$ is closed.

In this paper, we use the expression and the stability characterization of the Moore-Penrose inverse to investigate the condition for the perturbed operators to have the Hyers-Ulam stability and the condition in order that the Hyers-Ulam stability constant be continuous. In Section 2, we first establish a relationship between the Hyers-Ulam stability and the Moore-Penrose inverse, that is, a closed operator has the Hyers-Ulam stability if and only if it has the bounded Moore-Penrose 
inverse. Meanwhile, the stability constant is determined in terms of the MoorePenrose inverse. Utilizing this result, we give some sufficient conditions for the perturbed operators having the Hyers-Ulam stability and give an explicit expression of the Hyers-Ulam stability constant. In the case of bounded linear operators, some sufficient and necessary conditions for the Hyers-Ulam stability constants to be continuous are also provided in Section 3. In the end, as an application, we give a characterization for the Hyers-Ulam stability constants of the semi-Fredholm operators to be continuous.

\section{The CASE OF CLOSED LinEAR OPERATORS}

The following lemma is proved by a straightforward verification of the conditions of the definition of Moore-Penrose inverse.

Lemma 2.1. [12] Let $X, Y$ be Hilbert spaces and $T \in C(X, Y)$ with a generalized inverse $T^{+} \in B(Y, X)$, then $T$ has the bounded Moore-Penrose inverse $T^{\dagger}$ and

$$
T^{\dagger}=\left[I-P_{N(T)}^{\perp}\right] T^{+} P_{R(T)}^{\perp} .
$$

Theorem 2.2. Let $X, Y$ be Hilbert spaces and $T \in C(X, Y)$. Then the following statements are equivalent:

(1) T has the Hyers-Ulam stability;

(2) $T$ has the bounded Moore-Penrose inverse $T^{\dagger}$;

(3) $T$ has a bounded generalized inverse $T^{+}$;

(4) $T$ has closed range.

Moreover, if one of the conditions above is true, then $D\left(T^{\dagger}\right)=Y, N\left(T^{\dagger}\right)=$ $R(T)^{\perp}, R\left(T^{\dagger}\right)=D(T) \cap N(T)^{\perp}$ and

$$
K_{T}=\left\|T^{\dagger}\right\|=\gamma(T)^{-1}
$$

Proof. Note that $\left.T\right|_{N(T)^{\perp}}: D(T) \cap N(T)^{\perp} \rightarrow R(T)$ is invertible and $T^{\dagger}$ is defined by

$$
T^{\dagger} y=\left(\left.T\right|_{N(T)^{\perp}}\right)^{-1} Q y \quad\left(y \in R(T)+R(T)^{\perp}\right),
$$

where $Q$ is the orthogonal projector of $Y$ onto $\overline{R(T)}$ along $R(T)^{\perp}$. Then $T^{\dagger}$ is a densely defined closed operator with $D\left(T^{\dagger}\right)=R(T)+R(T)^{\perp}$ and $R\left(T^{\dagger}\right)=D(T) \cap$ $N(T)^{\perp}$.

$(2) \Rightarrow(1)$. If $T$ has the bounded Moore-Penrose inverse $T^{\dagger}$, then for all $x \in D(T)$, $\left(I-T^{\dagger} T\right) x \in N(T)$ and

$$
\left\|x-\left(I-T^{\dagger} T\right) x\right\|=\left\|T^{\dagger} T x\right\| \leq\left\|T^{\dagger}\right\|\|T x\| .
$$


This means that $T$ has the Hyers-Ulam stability and $K_{T} \leq\left\|T^{\dagger}\right\|$. Assume that $K$ is a Hyers-Ulam stability constant for $T$, i.e., for all $x \in D(T)$, there exists $x_{0} \in N(T)$ such that $\left\|x-x_{0}\right\| \leq K\|T x\|$. Then for all $y \in Y, T^{\dagger} y \in D(T) \cap N(T)^{\perp}$ and there exists $x_{1} \in N(T)$ such that $\left\|T^{\dagger} y-x_{1}\right\| \leq K\left\|T T^{\dagger} y\right\|$. Since $T^{\dagger} y \perp x_{1}$, we get

$$
\left\|T^{\dagger} y\right\| \leq\left\|T^{\dagger} y-x_{1}\right\| \leq K\left\|T T^{\dagger} y\right\| \leq K\|y\| .
$$

Hence $K \geq\left\|T^{\dagger}\right\|$ and thus $K_{T} \geq\left\|T^{\dagger}\right\|$. Therefore $K_{T}=\left\|T^{\dagger}\right\|$.

$(1) \Rightarrow(2)$. If $T$ has the Hyers-Ulam stability, then by (2.1), we know that the Moore-Penrose inverse $T^{\dagger}$ is bounded.

$(2) \Leftrightarrow(3)$. From Lemma 2.1, we can see $(3) \Rightarrow(2)$. Since the Moore-Penrose inverse $T^{\dagger}$ is also a generalized inverse, we can get $(2) \Rightarrow(3)$.

$(4) \Rightarrow(2)$. If $R(T)$ is closed, then $D\left(T^{\dagger}\right)=Y$ and by the Closed Graph Theorem, $T^{\dagger}$ is bounded.

(2) $\Rightarrow(4)$. If $T^{\dagger}$ is bounded, since $T^{\dagger}$ is a densely defined closed operator, we get $D\left(T^{\dagger}\right)=Y$, i.e, $Y=R(T)+R(T)^{\perp}$. This implies that $R(T)$ is closed. In the following, we shall show $\gamma(T)=\left\|T^{\dagger}\right\|^{-1}$. In fact, for all $x \in D(T)$, we have $\left(I-T^{\dagger} T\right) x \in N(T)$ and

$$
d(x, N(T)) \leq\left\|x-\left(I-T^{\dagger} T\right) x\right\|=\left\|T^{\dagger} T x\right\| \leq\left\|T^{\dagger}\right\|\|T x\| .
$$

Then $\gamma(T) \geq\left\|T^{\dagger}\right\|^{-1}$. Since $\gamma(T) \leq\|T x\|$ for all $x \in D(T) \cap N(T)^{\perp}$ with $\|x\|=1$, we get for all $y \in Y$ satisfying $\left\|T^{\dagger} y\right\|=1$,

$$
\gamma(T) \leq\left\|T T^{\dagger} y\right\|=\|Q y\| \leq\|y\| .
$$

Hence for all $y \in Y$ with $T^{\dagger} y \neq 0, \gamma(T) \leq \frac{\|y\|}{\left\|T^{\dagger} y\right\|}$. Thus

$$
\gamma(T) \leq \inf \left\{\frac{\|y\|}{\left\|T^{\dagger} y\right\|}: y \in Y, T^{\dagger} y \neq 0\right\}=\left(\sup \left\{\frac{\left\|T^{\dagger} y\right\|}{\|y\|}: y \in Y\right\}\right)^{-1}=\left\|T^{\dagger}\right\|^{-1}
$$

Therefore $\gamma(T)=\left\|T^{\dagger}\right\|^{-1}$.

Applying Theorem 2.2, we get [9, Theorem 3.1].

Corollary 2.3. [9] Let $X, Y$ be Hilbert spaces and $T \in C(X, Y)$. Then $T$ has the Hyers-Ulam stability if and only if $T$ has closed range. Moreover, in this case, $K_{T}=\gamma(T)^{-1}$.

In the following, we shall use the expressions and stability characterizations of the Moore-Penrose inverse to investigate the Hyers-Ulam stability of closed operators. 
We need the following lemma, which can be proved by using the fact that $P_{M} P_{M}^{*}[I-$ $\left.\left(P_{M}-P_{M}^{*}\right)^{2}\right]=\left[I-\left(P_{M}-P_{M}^{*}\right)^{2}\right] P_{M} P_{M}^{*}$.

Lemma 2.4. [12] Let $X$ be a Hilbert space and $M$ be a closed linear subspace of $X$. Let $P_{M}: X \rightarrow M$ be a (not necessarily selfadjoint) projector from $X$ onto $M$, then the orthogonal projector $P_{M}^{\perp}$ from $X$ onto $M$ can be expressed by

$$
P_{M}^{\perp}=P_{M} P_{M}^{*}\left[I-\left(P_{M}-P_{M}^{*}\right)^{2}\right]^{-1}=\left[I-\left(P_{M}-P_{M}^{*}\right)^{2}\right]^{-1} P_{M} P_{M}^{*} .
$$

Utilizing Lemma 2.1 with Lemma 2.4, we can get the following lemma.

Lemma 2.5. Let $T \in C(X, Y)$ with a bounded generalized inverse $T^{+} \in B(Y, X)$, then $T$ has the bounded Moore-Penrose inverse $T^{\dagger}$ and

$$
T^{\dagger}=\left\{I-\left[\left(T^{+} T\right)^{* *}-\left(T^{+} T\right)^{*}\right]^{2}\right\}^{-1}\left(T^{+} T\right)^{*} T^{+}\left(T T^{+}\right)^{*}\left\{I-\left[T T^{+}-\left(T T^{+}\right)^{*}\right]^{2}\right\}^{-1}
$$

Proof. Since $T T^{+}$is a projector from $Y$ onto $R(T)$, it follows from Lemma 2.4 that

$$
P_{R(T)}^{\perp}=T T^{+}\left(T T^{+}\right)^{*}\left\{I-\left[T T^{+}-\left(T T^{+}\right)^{*}\right]^{2}\right\}^{-1} .
$$

Noting that $I-T^{+} T$ is a bounded projector from $D(T)$ onto $N(T)$ and $D(T)$ is dense in $X$, one can verify that $\left(I-T^{+} T\right)^{*}$ is defined on the whole space $Y$. It follows from the Closed Graph Theorem that $\left(I-T^{+} T\right)^{*}$ is bounded. Hence $P_{N(T)}=\left(I-T^{+} T\right)^{* *}$, which is exactly the unique norm-preserving extension to whole space $X$ of $I-T^{+} T$. Thus $P_{N(T)}^{*}=\left(I-T^{+} T\right)^{*}$ and

$$
\begin{aligned}
& I-P_{N(T)}^{\perp} \\
= & I-\left[I-\left(P_{N(T)}-P_{N(T)}^{*}\right)^{2}\right]^{-1} P_{N(T)} P_{N(T)}^{*} \\
= & {\left[I-\left(P_{N(T)}-P_{N(T)}^{*}\right)^{2}\right]^{-1}\left[I-\left(P_{N(T)}-P_{N(T)}^{*}\right)^{2}-P_{N(T)} P_{N(T)}^{*}\right] } \\
= & {\left[I-\left(P_{N(T)}-P_{N(T)}^{*}\right)^{2}\right]^{-1}\left(I-P_{N(T)}^{*}\right)\left(I-P_{N(T)}\right) } \\
= & \left\{I-\left[\left(I-T^{+} T\right)^{* *}-\left(I-T^{+} T\right)^{*}\right]^{2}\right\}^{-1}\left[I-\left(I-T^{+} T\right)^{*}\right]\left[I-P_{N(T)}\right] \\
= & \left\{I-\left[\left(T^{+} T\right)^{* *}-\left(T^{+} T\right)^{*}\right]^{2}\right\}^{-1}\left(T^{+} T\right)^{*}\left[I-P_{N(T)}\right] .
\end{aligned}
$$

Therefore, by $\left.P_{N(T)}\right|_{D(T)}=I-T^{+} T$ and $R\left(T^{+}\right) \subset D(T)$, we obtain

$$
\begin{aligned}
T^{\dagger}= & {\left[I-P_{N(T)}^{\perp}\right] T^{+} P_{R(T)}^{\perp} } \\
= & \left\{I-\left[\left(T^{+} T\right)^{* *}-\left(T^{+} T\right)^{*}\right]^{2}\right\}^{-1}\left(T^{+} T\right)^{*}\left[I-P_{N(T)}\right] \\
& T^{+}\left(T T^{+}\right)\left(T T^{+}\right)^{*}\left\{I-\left[T T^{+}-\left(T T^{+}\right)^{*}\right]^{2}\right\}^{-1} \\
= & \left\{I-\left[\left(T^{+} T\right)^{* *}-\left(T^{+} T\right)^{*}\right]^{2}\right\}^{-1}\left(T^{+} T\right)^{*} T^{+}\left(T T^{+}\right)^{*}\left\{I-\left[T T^{+}-\left(T T^{+}\right)^{*}\right]^{2}\right\}^{-1} .
\end{aligned}
$$


Lemma 2.6. Let $T \in C(X, Y)$ have a generalized inverse $T^{+} \in B(Y, X)$ and $\delta T \in$ $L(X, Y)$ be $T$-bounded with constants $a, b$. If $a\left\|T^{+}\right\|+b\left\|T T^{+}\right\|<1$, then $\bar{T}=T+\delta T$ is closed and

$$
B=T^{+}\left(I+\delta T T^{+}\right)^{-1}: Y \rightarrow X
$$

satisfies $B \bar{T} B=B$ on $Y, R(B)=R\left(T^{+}\right)$and $N(B)=N\left(T^{+}\right)$.

Proof. It follows from Theorem 1.10 that $\bar{T}$ is closed. Since

$$
\begin{aligned}
\left\|\delta T T^{+} y\right\| & \leq a\left\|T^{+} y\right\|+b\left\|T T^{+} y\right\| \\
& \leq\left(a\left\|T^{+}\right\|+b\left\|T T^{+}\right\|\right)\|y\|,
\end{aligned}
$$

for all $y \in Y$, we get $\left\|\delta T T^{+}\right\| \leq a\left\|T^{+}\right\|+b\left\|T T^{+}\right\|<1$. By the celebrated Banach Lemma, the inverse of $I+\delta T T^{+}$exists and $\left(I+\delta T T^{+}\right)^{-1} \in B(Y)$. Hence $B=T^{+}(I+$ $\left.\delta T T^{+}\right)^{-1}: Y \rightarrow X$ is a bounded linear operator. It is easy to verify $R(B)=R\left(T^{+}\right)$ and $N(B)=N\left(T^{+}\right)$. To the end, we need to show $B \bar{T} B=B$ on $Y$. Indeed, $R(B)=R\left(T^{+}\right) \subseteq D(T)=D(\bar{T})$. Since

$$
\left(I+\delta T T^{+}\right) T T^{+}=T T^{+}+\delta T T^{+}=(T+\delta T) T^{+}=\bar{T} T^{+},
$$

we have $T T^{+}=\left(I+\delta T T^{+}\right)^{-1} \bar{T} T^{+}$and therefore,

$$
\begin{aligned}
B \bar{T} B & =T^{+}\left(I+\delta T T^{+}\right)^{-1} \bar{T} T^{+}\left(I+\delta T T^{+}\right)^{-1} \\
& =T^{+} T T^{+}\left(I+\delta T T^{+}\right)^{-1}=T^{+}\left(I+\delta T T^{+}\right)^{-1}=B .
\end{aligned}
$$

In the next theorem some conditions are given for $\bar{T}$ to have the Hyers-Ulam stability as well as the Hyers-Ulam stability constant $K_{\bar{T}}$ is explicitly expressed.

Theorem 2.7. Let $X, Y$ be Hilbert spaces and let $T \in C(X, Y)$ have the HyersUlam stability. Let $T^{+} \in B(Y, X)$ be a bounded generalized inverse of $T$ and $\delta T \in$ $L(X, Y)$ be $T$-bounded with constants $a, b$. If $a\left\|T^{+}\right\|+b\left\|T T^{+}\right\|<1$, then the following statements are equivalent:

(1) $B=T^{+}\left(I+\delta T T^{+}\right)^{-1}: Y \rightarrow X$ is a bounded generalized inverse of $\bar{T}=T+\delta T$;

(2) $\left(I+\delta T T^{+}\right)^{-1} R(\bar{T})=R(T)$;

(3) $\left(I+\delta T T^{+}\right)^{-1} \bar{T}$ maps $N(T)$ into $R(T)$;

(4) $R(\bar{T}) \cap N\left(T^{+}\right)=\{0\}$. 
Moreover, if one of the conditions above is true, then $R(\bar{T})$ is closed, $\bar{T}$ has the Hyers-Ulam stability and $K_{\bar{T}}=\left\|\bar{T}^{\dagger}\right\|$, where

$$
\begin{aligned}
\bar{T}^{\dagger}= & \left\{I-\left[\left(T^{+}\left(I+\delta T T^{+}\right)^{-1} \bar{T}\right)^{* *}-\left(T^{+}\left(I+\delta T T^{+}\right)^{-1} \bar{T}\right)^{*}\right]^{2}\right\}^{-1} \\
& {\left[T^{+}\left(I+\delta T T^{+}\right)^{-1} \bar{T}\right]^{*} T^{+}\left(I+\delta T T^{+}\right)^{-1}\left[\bar{T} T^{+}\left(I+\delta T T^{+}\right)^{-1}\right]^{*} } \\
& \left\{I-\left[\bar{T} T^{+}\left(I+\delta T T^{+}\right)^{-1}-\left(\bar{T} T^{+}\left(I+\delta T T^{+}\right)^{-1}\right)^{*}\right]^{2}\right\}^{-1} .
\end{aligned}
$$

Proof. From Theorem 1.10, we can see the equivalence. If one of the conditions is true, then $R(\bar{T})$ is closed and $T^{+}\left(I+\delta T T^{+}\right)^{-1}: Y \rightarrow X$ is a bounded generalized inverse of $T+\delta T$. By Lemma 2.5, we can get what we desired.

Remark 2.8. It should be noted that under any one of the conditions in Theorem 2.7, the Hyers-Ulam stability constant $K_{\bar{T}}$ is continuous at $T$ if $\delta T$ is bounded and $\|\delta T\| \rightarrow 0$. In fact,

$$
\begin{aligned}
& T^{+}\left(I+\delta T T^{+}\right)^{-1} \bar{T}=\left(I+T^{+} \delta T\right)^{-1} T^{+} \bar{T} \\
= & \left(I+T^{+} \delta T\right)^{-1} T^{+} T+\left(I+T^{+} \delta T\right)^{-1} T^{+} \delta T \rightarrow T^{+} T
\end{aligned}
$$

and

$$
\begin{aligned}
& \bar{T} T^{+}\left(I+\delta T T^{+}\right)^{-1} \\
= & T T^{+}\left(I+\delta T T^{+}\right)^{-1}+\delta T T^{+}\left(I+\delta T T^{+}\right)^{-1} \rightarrow T T^{+},
\end{aligned}
$$

as $\|\delta T\| \rightarrow 0$.

Corollary 2.9. Let $X, Y$ be Hilbert spaces and let $T \in C(X, Y)$ have the HyersUlam stability. Let $T^{+} \in B(Y, X)$ be a bounded generalized inverse of $T$ and $\delta T \in$ $L(X, Y)$ be $T$-bounded with constants $a, b$. If $a\left\|T^{+}\right\|+b\left\|T T^{+}\right\|<1$ and $N(T) \subseteq$ $N(\delta T)$, then $\bar{T}=T+\delta T$ has the Hyers-Ulam stability and $K_{\bar{T}}=\left\|\bar{T}^{\dagger}\right\|$, where

$$
\begin{aligned}
\bar{T}^{\dagger}= & \left\{I-\left[\left(T^{+} T\right)^{* *}-\left(T^{+} T\right)^{*}\right]^{2}\right\}^{-1}\left(T^{+} T\right)^{*} T^{+}\left(I+\delta T T^{+}\right)^{-1}\left[\bar{T} T^{+}\left(I+\delta T T^{+}\right)^{-1}\right]^{*} \\
& \left\{I-\left[\bar{T} T^{+}\left(I+\delta T T^{+}\right)^{-1}-\left(\left(\bar{T} T^{+}\left(I+\delta T T^{+}\right)^{-1}\right)^{*}\right]^{2}\right\}^{-1} .\right.
\end{aligned}
$$

Proof. We first show that $N(\bar{T})=N(T)$. In fact, by $N(T) \subseteq N(\delta T)$, we have $N(T) \subseteq N(\bar{T})$ as well as $\delta T\left(I-T^{+} T\right)=0$, whence $\delta T=\delta T T^{+} T$. If $x \in N(\bar{T})$, then

$$
0=\bar{T} x=T x+\delta T x=T x+\delta T T^{+} T x=\left(I+\delta T T^{+}\right) T x .
$$

Since $I+\delta T T^{+}$is invertible, $T x=0$. Hence $N(\bar{T})=N(T)$. Thus $P_{N(\bar{T})}^{\perp}=P_{N(T)}^{\perp}$ and $\left.P_{N(\bar{T})}^{\perp}\right|_{D(T)}=I-\left\{I-\left[\left(I-T^{+} T\right)^{* *}-\left(I-T^{+} T\right)^{*}\right]^{2}\right\}^{-1}\left[I-\left(I-T^{+} T\right)^{*}\right] T^{+} T$. Therefore, by Lemma 2.5, we can get what we desired. 
Corollary 2.10. Let $X, Y$ be Hilbert spaces and let $T \in C(X, Y)$ have the HyersUlam stability. Let $T^{+} \in B(Y, X)$ be a bounded generalized inverse of $T$ and $\delta T \in$ $L(X, Y)$ be $T$-bounded with constants $a, b$. If $a\left\|T^{+}\right\|+b\left\|T T^{+}\right\|<1$ and $R(\delta T) \subseteq$ $R(T)$, then $\bar{T}=T+\delta T$ has the Hyers-Ulam stability and $K_{\bar{T}}=\left\|\bar{T}^{\dagger}\right\|$, where

$$
\begin{aligned}
\bar{T}^{\dagger}= & \left\{I-\left[\left(T^{+}\left(I+\delta T T^{+}\right)^{-1} \bar{T}\right)^{* *}-\left(T^{+}\left(I+\delta T T^{+}\right)^{-1} \bar{T}\right)^{*}\right]^{2}\right\}^{-1} \\
& {\left[T^{+}\left(I+\delta T T^{+}\right)^{-1} \bar{T}\right]^{*} T^{+}\left(I+\delta T T^{+}\right)^{-1}\left(T T^{+}\right)^{*}\left\{I-\left[T T^{+}-\left(T T^{+}\right)^{*}\right]^{2}\right\}^{-1} . }
\end{aligned}
$$

Proof. We first show $R(\bar{T})=R(T)$. In fact, by $R(\delta T) \subseteq R(T)$, we have $R(\bar{T}) \subseteq$ $R(T)$ and also $\left(I-T T^{+}\right) \delta T=0$, which implies $\delta T=T T^{+} \delta T$. Then

$$
\bar{T} T^{+}=(T+\delta T) T^{+}=T T^{+}+T T^{+} \delta T T^{+}=T T^{+}\left(I+\delta T T^{+}\right)
$$

and $\bar{T} T^{+}\left(I+\delta T T^{+}\right)^{-1}=T T^{+}$. Hence $T=T T^{+} T=\bar{T} T^{+}\left(I+\delta T T^{+}\right)^{-1} T$. This means $R(T) \subseteq R(\bar{T})$. Thus $R(\bar{T})=R(T)$ and

$$
P_{R(\bar{T})}^{\perp}=P_{R(T)}^{\perp}=T T^{+}\left(T T^{+}\right)^{*}\left\{I-\left[T T^{+}-\left(T T^{+}\right)^{*}\right]^{2}\right\}^{-1} .
$$

By $R\left(I-T T^{+}\right)=N\left(T^{+}\right)=N(B)$, we have $T^{+}\left(I+\delta T T^{+}\right)^{-1} T T^{+}=T^{+}\left(I+\delta T T^{+}\right)^{-1}$. Therefore by Lemma 2.5, we can get what we desired.

Remark 2.11. It should be noted that if $N(T) \subseteq N(\delta T)$ or $R(\delta T) \subseteq R(T)$ holds, then the Hyers-Ulam stability constant $K_{\bar{T}}$ is continuous at $T$ as $\delta T$ is bounded and $\|\delta T\| \rightarrow 0$.

Recall that a closed operator $T: X \rightarrow Y$ is called left semi-Fredholm if $\operatorname{dim} N(T)<$ $\infty$ and $R(T)$ is closed. It is called right semi-Fredholm if $\operatorname{codim} R(T)<\infty$ and $R(T)$ is closed. We say a closed operator $T$ is semi-Fredholm if it is left or right semi-Fredholm. The following result involving semi-Fredholm operators holds.

Theorem 2.12. Let $X, Y$ be Hilbert spaces and let $T \in C(X, Y)$ be a semi-Fredholm operator. Let $T^{+} \in B(Y, X)$ be a bounded generalized inverse of $T$ and $\delta T \in L(X, Y)$ be $T$-bounded with constants $a, b$. If $a\left\|T^{+}\right\|+b\left\|T T^{+}\right\|<1$ and

either $\operatorname{dim} N(\bar{T})=\operatorname{dim} N(T)<\infty \quad$ or $\quad \operatorname{codim} R(\bar{T})=\operatorname{codim} R(T)<\infty$, then $\bar{T}=T+\delta T$ has the Hyers-Ulam stability and $K_{\bar{T}}=\left\|\bar{T}^{\dagger}\right\|$, where

$$
\begin{aligned}
\bar{T}^{\dagger}= & \left\{I-\left[\left(T^{+}\left(I+\delta T T^{+}\right)^{-1} \bar{T}\right)^{* *}-\left(T^{+}\left(I+\delta T T^{+}\right)^{-1}(T+\delta T)\right)^{*}\right]^{2}\right\}^{-1} \\
& {\left[T^{+}\left(I+\delta T T^{+}\right)^{-1} \bar{T}\right]^{*} T^{+}\left(I+\delta T T^{+}\right)^{-1}\left[\bar{T} T^{+}\left(I+\delta T T^{+}\right)^{-1}\right]^{*} } \\
& \left\{I-\left[\bar{T} T^{+}\left(I+\delta T T^{+}\right)^{-1}-\left(\bar{T} T^{+}\left(I+\delta T T^{+}\right)^{-1}\right)^{*}\right]^{2}\right\}^{-1} .
\end{aligned}
$$


Proof. By Lemma 2.6, $B=B \bar{T} B$ on $Y$, where $B=T^{+}\left(I+\delta T T^{+}\right)^{-1}$. Then $B \bar{T} B \bar{T}=$ $B \bar{T}$ and it follows that $B \bar{T}$ is an idempotent on $D(\bar{T})$. Due to $\bar{T}\left(I-T^{+} T\right)=$ $(T+\delta T)\left(I-T^{+} T\right)=\delta T\left(I-T^{+} T\right)$ and

$$
\begin{aligned}
\left\|\left[\delta T\left(I-T^{+} T\right)\right] x\right\| & \leq a\left\|\left(I-T^{+} T\right) x\right\|+b\left\|T\left(I-T^{+} T\right) x\right\| \\
& \leq a\left\|I-T^{+} T\right\| \cdot\|x\| \quad(x \in D(T))
\end{aligned}
$$

we conclude that

$$
\begin{aligned}
B \bar{T} & =T^{+}\left[I+(\bar{T}-T) T^{+}\right]^{-1} \bar{T} \\
& =T^{+}\left[I+(\bar{T}-T) T^{+}\right]^{-1} \bar{T} T^{+} T+T^{+}\left[I+(\bar{T}-T) T^{+}\right]^{-1} \bar{T}\left(I-T^{+} T\right) \\
& =T^{+} T+T^{+}\left[I+(\bar{T}-T) T^{+}\right]^{-1} \bar{T}\left(I-T^{+} T\right)
\end{aligned}
$$

is bounded. Hence $B \bar{T}$ can be extended uniquely from $D(\bar{T})$ to $X$. We denote its extension by $S$, which is defined by $S x=\lim _{n \rightarrow+\infty} B \bar{T} x_{n}$ for all $x \in X$, where $x_{n} \in D(\bar{T})$ satisfies $x_{n} \rightarrow x$. It is easy to verify $S \in B(X)$ and $S^{2}=S$. Since $R(B \bar{T}) \subset R(S) \subset \overline{R(B \bar{T})}$, we get $R(S)=\overline{R(B \bar{T})}=\overline{R(B)}=\overline{R\left(T^{+}\right)}$. Next, for any $x \in N(S)$, then there exists $\left\{x_{n}\right\} \subset D(\bar{T})$ with $x_{n} \rightarrow x$ such that $B \bar{T} x_{n} \rightarrow S x=0$. Hence $x_{n}-B \bar{T} x_{n} \in N(B \bar{T})$ satisfies $x_{n}-B \bar{T} x_{n} \rightarrow x$, which implies $N(S) \subset \overline{N(B \bar{T})}$. Because $N(B \bar{T}) \subset N(S)$ and $N(S)$ is closed, $\overline{N(B \bar{T})} \subset N(S)$. Thus we conclude $N(S)=\overline{N(B \bar{T})}$. On the other hand, by the Closed Graph Theorem, we can observe that $\bar{T} B$ is a projector from $Y$ onto $R(\bar{T} B)$ and $N(\bar{T} B)=N(B)=N\left(T^{+}\right)$. Thus by $X=R(S) \oplus N(S)$ and $Y=R(\bar{T} B) \oplus N(B)$, we get

$$
\overline{R\left(T^{+}\right)} \oplus N(T)=X=\overline{R(B \bar{T})} \oplus \overline{N(B \bar{T})}=\overline{R\left(T^{+}\right)} \oplus \overline{N(B \bar{T})}
$$

and

$$
R(T) \oplus N\left(T^{+}\right)=Y=R(\bar{T} B) \oplus N\left(T^{+}\right)=R(\bar{T})+N\left(T^{+}\right)=R(\bar{T}) \oplus N^{-},
$$

where $N^{-}$satisfies $N\left(T^{+}\right)=N^{-} \oplus\left(R(\bar{T}) \cap N\left(T^{+}\right)\right)$. If $\operatorname{dim} N(\bar{T})=\operatorname{dim} N(T)<\infty$, then by (2.2), we get $\operatorname{dim} \overline{N(B \bar{T})}=\operatorname{dim} N(\bar{T})$. Noting $N(\bar{T}) \subseteq \overline{N(B \bar{T})}$, we can obtain $N(\bar{T})=\overline{N(B \bar{T})}$. Hence for any $y \in R(\bar{T}) \cap N\left(T^{+}\right)$, there exists $x \in D(\bar{T})$ such that $y=\bar{T} x$ and $T^{+} \bar{T} x=0$. This means $\bar{T} x \in N\left(T^{+}\right)=N(B)$. Thus $x \in N(B \bar{T})$ and so $x \in N(\bar{T})$, which implies $y=\bar{T} x=0$. Therefore $R(\bar{T}) \cap N\left(T^{+}\right)=$ $\{0\}$. If $\operatorname{codim} R(\bar{T})=\operatorname{codim} R(T)<\infty$, then by $(2.3), \operatorname{dim} N\left(T^{+}\right)=\operatorname{dim} N^{-}$and so $R(\bar{T}) \cap N\left(T^{+}\right)=\{0\}$. Using Theorem 2.7 we can get what we desired. 


\section{THE CASE OF BOUNDED LINEAR OPERATORS}

In this section, we shall give some sufficient and necessary conditions for the Hyers-Ulam stability constants to be continuous.

Theorem 3.1. Let $X, Y$ be Hilbert spaces and let $T \in B(X, Y)$ have the HyersUlam stability, i.e, $T$ has a Moore-Penrose inverse $T^{\dagger} \in B(Y, X)$. If $\delta T \in B(X, Y)$ satisfies $\|\delta T\|\left\|T^{\dagger}\right\|<1$, then the following statements are equivalent:

(1) $B=T^{\dagger}\left(I+\delta T T^{\dagger}\right)^{-1}: Y \rightarrow X$ is a bounded generalized inverse of $\bar{T}=T+\delta T$;

(2) $\left(I+\delta T T^{\dagger}\right)^{-1} R(\bar{T})=R(T)$;

(3) $\left(I+\delta T T^{\dagger}\right)^{-1} \bar{T}$ maps $N(T)$ into $R(T)$;

(4) $\left(I+T^{\dagger} \delta T\right)^{-1} N(T)=N(\bar{T})$;

(5) $R(\bar{T}) \cap N\left(T^{\dagger}\right)=\{0\}$;

(6) $\bar{T}$ has the Hyers-Ulam stability and the Hyers-Ulam stability constant $K_{\bar{T}}$ satisfies

$$
\lim _{\|\delta T\| \rightarrow 0} K_{\bar{T}}=K_{T}
$$

(7) $\bar{T}$ has the Hyers-Ulam stability and there exist $M>0$ and $\varepsilon>0$ such that $K_{\bar{T}} \leq M$ for all $\|\delta T\|<\varepsilon$;

(8) $\bar{T}$ has the bounded Moore-Penrose inverse $\bar{T}^{\dagger} \in B(Y, X)$ with $\lim _{\|\delta T\| \rightarrow 0} \bar{T}^{\dagger}=T^{\dagger}$.

In this case, $K_{\bar{T}}=\left\|\bar{T}^{\dagger}\right\|$ and

$$
\begin{aligned}
\bar{T}^{\dagger}= & \left\{I-\left[T^{+}\left(I+\delta T T^{+}\right)^{-1} \bar{T}-\left(T^{+}\left(I+\delta T T^{+}\right)^{-1} \bar{T}\right)^{*}\right]^{2}\right\}^{-1} \\
& {\left[T^{+}\left(I+\delta T T^{+}\right)^{-1} \bar{T}\right]^{*} T^{+}\left(I+\delta T T^{+}\right)^{-1}\left[\bar{T} T^{+}\left(I+\delta T T^{+}\right)^{-1}\right]^{*} } \\
& \left\{I-\left[\bar{T} T^{+}\left(I+\delta T T^{+}\right)^{-1}-\left(\bar{T} T^{+}\left(I+\delta T T^{+}\right)^{-1}\right)^{*}\right]^{2}\right\}^{-1} .
\end{aligned}
$$

Proof. In Theorem 2.7, we take $a=\|\delta T\|$ and $b=0$. Then it follows that (1), (2), (3) and (5) are equivalent. The equivalence of (4) and (5) can be found in [11]. By Theorem 2.2 and Theorem 2.7, we can see $(5) \Rightarrow(8)$ and $(8) \Rightarrow(6)$. Obviously, $(6) \Rightarrow(7)$. Next we shall show $(7) \Rightarrow(8)$ and $(8) \Rightarrow(5)$. Since

$$
\begin{aligned}
& \bar{T}^{\dagger}-T^{\dagger} \\
= & \bar{T}^{\dagger}\left(I-T T^{\dagger}\right)+\bar{T}^{\dagger}(T-\bar{T}) T^{\dagger}+\left(\bar{T}^{\dagger} \bar{T}-I\right) T^{\dagger} \\
= & \bar{T}^{\dagger} \bar{T} \bar{T}^{\dagger}\left(I-T T^{\dagger}\right)+\bar{T}^{\dagger}(T-\bar{T}) T^{\dagger}+\left(\bar{T}^{\dagger} \bar{T}-I\right) T^{\dagger} T T^{\dagger} \\
= & \bar{T}^{\dagger}\left[\left(I-T T^{\dagger}\right) \bar{T} \bar{T}^{\dagger}\right]^{*}+\bar{T}^{\dagger}(T-\bar{T}) T^{\dagger}+\left[T^{\dagger} T\left(\bar{T}^{\dagger} \bar{T}-I\right)\right]^{*} T^{\dagger} \\
= & \bar{T}^{\dagger}\left[\left(I-T T^{\dagger}\right)(\bar{T}-T) \bar{T}^{\dagger}\right]^{*}+\bar{T}^{\dagger}(T-\bar{T}) T^{\dagger}+\left[T^{\dagger}(T-\bar{T})\left(\bar{T}^{\dagger} \bar{T}-I\right)\right]^{*} T^{\dagger} \\
= & \bar{T}^{\dagger}\left(\bar{T}^{\dagger}\right)^{*}(\bar{T}-T)^{*}\left(I-T T^{\dagger}\right)+\bar{T}^{\dagger}(T-\bar{T}) T^{\dagger}+\left(\bar{T}^{\dagger} \bar{T}-I\right)(T-\bar{T})^{*}\left(T^{\dagger}\right)^{*} T^{\dagger},
\end{aligned}
$$


we can get

$$
\left\|\bar{T}^{\dagger}-T^{\dagger}\right\| \leq\left(\left\|\bar{T}^{\dagger}\right\|^{2}+\left\|\bar{T}^{\dagger}\right\|\left\|T^{\dagger}\right\|+\left\|T^{\dagger}\right\|^{2}\right)\|\delta T\| \text {. }
$$

Combining it with $\left\|\bar{T}^{\dagger}\right\|=K_{\bar{T}} \leq M$, we can conclude that $\lim _{\|\delta T\| \rightarrow 0} \bar{T}^{\dagger}=T^{\dagger}$. To the end, we only need to prove $(8) \Rightarrow(5)$. If $\bar{T}$ has the bounded Moore-Penrose inverse $\bar{T}^{\dagger}$ with $\lim _{\|\delta T\| \rightarrow 0} \bar{T}^{\dagger}=T^{\dagger}$, then $P_{N(\bar{T})}^{\perp}=I-\bar{T}^{\dagger} \bar{T}$ and $P_{N(T)}^{\perp}=I-T^{\dagger} T$ and $\lim _{\|\delta T\| \rightarrow 0} P_{N(\bar{T})}^{\perp}=P_{N(T)}^{\perp}$. Without loss of generality, we may assume $\left\|P_{N(\bar{T})}^{\perp}-P_{N(T)}^{\perp}\right\|<$ 1. Then

$$
\begin{aligned}
P_{N(T)}^{\perp} N(\bar{T}) & =\left(I-T^{\dagger} T\right) N(\bar{T}) \\
& =\left(I-T^{\dagger} \bar{T}+T^{\dagger} \delta T\right) N(\bar{T}) \\
& =\left(I+T^{\dagger} \delta T\right) N(\bar{T})
\end{aligned}
$$

and by the Banach Lemma, the operator

$$
W=I-P_{N(T)}^{\perp}+P_{N(\bar{T})}^{\perp} P_{N(T)}^{\perp}=I+\left(P_{N(\bar{T})}^{\perp}-P_{N(T)}^{\perp}\right) P_{N(T)}^{\perp}
$$

is invertible and its inverse $W^{-1}: X \rightarrow X$ is bounded. Take any $y \in R(\bar{T}) \cap N\left(T^{\dagger}\right)$, then $y=\bar{T} x$ and $T^{\dagger} \bar{T} x=0$, where $x \in X$. Hence

$$
T\left(I+T^{\dagger} \delta T\right) x=T\left(I+T^{\dagger} \bar{T}-T^{\dagger} T\right) x=0,
$$

which implies $\left(I+T^{\dagger} \delta T\right) x \in N(T)$. Therefore

$$
\begin{aligned}
\left(I+T^{\dagger} \delta T\right) x & =P_{N(T)}^{\perp}\left(I+T^{\dagger} \delta T\right) x \\
& =P_{N(T)}^{\perp} W W^{-1}\left(I+T^{\dagger} \delta T\right) x \\
& =P_{N(T)}^{\perp}\left(I-P_{N(T)}^{\perp}+P_{N(\bar{T})}^{\perp} P_{N(T)}^{\perp}\right) W^{-1}\left(I+T^{\dagger} \delta T\right) x \\
& =P_{N(T)}^{\perp} P_{N(\bar{T})}^{\perp} P_{N(T)}^{\perp} W^{-1}\left(I+T^{\dagger} \delta T\right) x \\
& \in P_{N(T)}^{\perp} N(\bar{T})=\left(I+T^{\dagger} \delta T\right) N(\bar{T})
\end{aligned}
$$

Since $T^{\dagger} \delta T+I$ is invertible, we get $y=\bar{T} x=0$. Thus we obtain (5).

Theorem 3.2. Let $X, Y$ be Hilbert spaces, $T \in B(X, Y)$ be a finite rank operator and $T^{\dagger} \in B(Y, X)$ be a Moore-Penrose inverse of $T$. If $\delta T \in B(X, Y)$ satisfies $\|\delta T\|\left\|T^{\dagger}\right\|<1$, then $\bar{T}=T+\delta T$ has the Hyers-Ulam stability and the Hyers-Ulam stability constant $K_{\bar{T}}$ satisfies $\lim _{\|\delta T\| \rightarrow 0} K_{\bar{T}}=K_{T}$ if and only if

$$
\operatorname{Rank} \bar{T}=\operatorname{Rank} T<+\infty \text {. }
$$


Proof. The necessity follows from $(6) \Rightarrow(2)$ in Theorem 3.1. Next, we shall show the sufficiency. By Lemma 2.5, $\bar{T} B$ is a projector from $Y$ onto $R(\bar{T} B)$. Then

$$
R(T) \dot{+} N\left(T^{\dagger}\right)=Y=R(\bar{T} B) \oplus N(B)=R(\bar{T} B) \oplus N\left(T^{\dagger}\right) .
$$

Noting $\operatorname{dim} R(T)=\operatorname{codim} N\left(T^{\dagger}\right)=\operatorname{dim} R(\bar{T} B)$, we have $\operatorname{dim} R(\bar{T})=\operatorname{dim} R(\bar{T} B)$, so $R(\bar{T} B)=R(\bar{T})$. Hence by $R(\bar{T} B) \cap N\left(T^{\dagger}\right)=R(\bar{T} B) \cap N(B)=\{0\}$, we get $R(\bar{T}) \cap N\left(T^{\dagger}\right)=\{0\}$.

Theorem 3.3. Let $X, Y$ be Hilbert spaces and let $T \in B(X, Y)$ be a semi-Fredholm operator. If $T$ has the bounded Moore-Penrose inverse $T^{\dagger} \in B(Y, X)$ and $\delta T \in$ $B(X, Y)$ satisfies $\|\delta T\|\left\|T^{\dagger}\right\|<1$, then $\bar{T}=T+\delta T$ has the Hyers-Ulam stability and the Hyers-Ulam stability constant $K_{\bar{T}}$ satisfies $\lim _{\|\delta T\| \rightarrow 0} K_{\bar{T}}=K_{T}$ if and only if

either $\operatorname{dim} N(\bar{T})=\operatorname{dim} N(T)<\infty$ or $\operatorname{codim} R(\bar{T})=\operatorname{codim} R(T)<\infty$.

Proof. It is easy to see that the sufficiency follows from Theorem 2.12. Next we shall prove the necessity. If $\operatorname{dim} N(T)<\infty$, then by $(6) \Rightarrow$ (4) in Theorem 3.1, we can see $\operatorname{dim} N(\bar{T})=\operatorname{dim} N(T)$. If $\operatorname{codim} R(T)<\infty$, then by Theorem 3.1, $T^{\dagger}\left(I+\delta T T^{\dagger}\right)^{-1}: Y \rightarrow X$ is a bounded generalized inverse of $T+\delta T$. Hence from Lemma 2.6, $N(B)=N\left(T^{\dagger}\right)$ and

$$
Y=R(T) \dot{+} N\left(T^{\dagger}\right)=R(\bar{T}) \oplus N(B)=R(\bar{T}) \oplus N\left(T^{\dagger}\right) .
$$

Thus $\operatorname{dim} N\left(T^{\dagger}\right)=\operatorname{codim} R(T)<\infty$ and therefore $\operatorname{codim} R(\bar{T})=\operatorname{codim} R(T)$.

\section{REFERENCES}

[1] T. Aoki, On the stability of the linear transformationin Banach spaces. J. Math. Soc. Japan 2: 64-66, 1950.

[2] C. Badea and M. Mbekhta, Generalized inverses and the maximal radius of regularity of a Fredholm operator. Integral Equations Operator Theory, 28: 133-146, 1997.

[3] A. Ben-Israel and T.N.E. Greville, Generalized Inverses: Theory and Applications. SpringerVerlag, New York, 2003.

[4] G. Chen and Y. Xue, Perturbation analysis for the operator equation $T x=b$ in Banach spaces. J. Math. Anal. Appl., 212: 107-125, 1997.

[5] J. Chmielin'ski, D. Ilisević, M.S. Moslehian and Gh. Sadeghi, Perturbation of the Wigner equation in inner product $C^{*}$-modules. J. Math. Phys. 49: 8 pp, 2008.

[6] S. Czerwik, Stability of Functional Equations of Ulam-Hyers-Rassias Type. Hadronic Press, Palm Harbor, Florida, 2003.

[7] H.G. Dales and M.S. Moslehian, Stability of mappings on multi-normed spaces. Glasgow Math. J., 49: 321-332, 2007. 
[8] J. Ding and L. Huang, On the continuity of generalized inverses of linear operators in Hilbert spaces. Linear Algebra Appl., 262: 229-242, 1997.

[9] G. Hirasawa and T. Miura, Hyers-Ulam Stability of a closed operator in a Hilbert space. Bull. Korean. Math. Soc., 43: 107-117, 2006.

[10] Q. Huang, On perturbations for oblique projection generalized inverses of closed linear operators in Banach spaces. Linear Algebra Appl., 434: 2468-2474, 2011.

[11] Q. Huang and J. Ma, Continuity of generalized inverses of linear operators in Banach spaces and its applications. Appl. Math. Mech., 26: 1657-1663, 2005.

[12] Q. Huang and W. Zhai, Perturbations and expressions for generalized inverses in Banach spaces and Moore-Penrose inverses in Hilbert spaces of closed linear operators. Linear Algebra Appl., 435: 117-127, 2011.

[13] D.H. Hyers, On the stability of the linear functional equation. Proc. Nat. Acad. Sci. U.S.A., 27: 222-224, 1941.

[14] D.H. Hyers, G. Isac and Th.M. Rassias, Stability of Functional Equations in Several Variables. Birkhäuser, Basel, 1998.

[15] S.M. Jung, Hyers-Ulam-Rassias Stability of Functional Equations in Nonlinear Analysis. Springer Optimization and Its Applications, Vol 48, Springer, 2011.

[16] T. Kato, Perturbation Theory for Linear Operators. Springer-Verlag, Berlin, 1984.

[17] T. Miura, S. Miyajima and S.-E. Takahasi, Hyers-Ulam stability of linear differential operator with constant coeficients. Math. Nachr., 258: 90-96, 2003.

[18] T. Miura, S. Miyajima and S.-E. Takahasi, A characterization of Hyers-Ulam stability of first order linear diffierential operators. J. Math. Anal. Appl. 286: 136-146, 2003.

[19] T. Miura, A. Uchiyama, H. Oka, G. Hirasawa, S.-E. Takahasi and N. Niwa, A perturbation of normal operators on a Hilbert space. Nonlinear Funct. Anal. Appl., 13: 291-297, 2008.

[20] M.S. Moslehian, Ternary derivations, stability and physical aspects. Acta Appl. Math., 100: 187-199, 2008.

[21] M.S. Moslehian and Gh. Sadeghi, Perturbation of closed range operators. Turkish J. Math., 33: 143-149, 2009.

[22] M.Z. Nashed (Ed.), Generalized Inverses and Applications. Academic Press, New York, 1976.

[23] J.M. Rassias, On approximation of approximately linear mappings by linear mappings. $J$. Funct. Anal., 46: 126-130, 1982.

[24] Th.M. Rassias, On the stability of the linear mapping in Banach spaces. Proc. Amer. Math. Soc., 72: 297-300, 1978.

[25] H. Takagi, T. Miura and S.-E. Takahasi, Essential norms and stability constants of weighted composition operators on $C(X)$. Bull. Korean Math. Soc., 40: 583-591, 2003.

[26] L. Tan and S. Xiang, On the Aleksandrov-Rassias problem and the Hyers-Ulam-Rassias stability problem. Banach J. Math. Anal., 1: 11-22, 2007.

[27] S.M. Ulam, A collection of mathematical problems, Interscience Tracts in Pure and Applied Mathematics. no. 8, Interscience Publishers, New York-London, 1960.

[28] G. Wang, Y. Wei and S. Qiao, Generalized Inverses: Theory and Computations. Science Press, Beijing, New York, 2004. 
[29] Y. Wang and H. Zhang, Perturbation analysis for oblique projection generalized inverses of closed linear operators in Banach spaces. Linear Algebra Appl., 426: 1-11, 2007.

${ }^{1}$ College of Mathematics, Yangzhou University, Yangzhou 225002, China; School of Mathematical Sciences, Monash University, ViC 3800, Australia

E-mail address: qlhmath@yahoo.com.cn

2 Department of Pure Mathematics, Ferdowsi University of Mashhad, P.O. Box 1159, MashHad 91775, Iran.

E-mail address: moslehian@um.ac.ir and moslehian@memebr.ams.org

URL: http://profsite.um.ac.ir/ moslehian/ 